\title{
A Short Travel for Neutrinos in Large Extra Dimensions
}

\section{G. V. Stenico* ${ }^{\dagger}$}

Instituto de Física Gleb Wataghin - UNICAMP, 13083-859, Campinas, SP, Brazil

E-mail: gstenicoeifi.unicamp.br

D. V. Forero $\ddagger$

Instituto de Física Gleb Wataghin - UNICAMP, 13083-859, Campinas, SP, Brazil

E-mail: dvanegaseifi.unicamp.br

\section{O. L. G. Peres ${ }^{\S}$}

Instituto de Física Gleb Wataghin - UNICAMP, 13083-859, Campinas, SP, Brazil

E-mail: orlandodifi.unicamp.br

\begin{abstract}
Neutrino oscillations successfully explain the flavor transitions observed in neutrinos produced in natural sources like the center of the sun and the earth atmosphere, and also from man-made sources like reactors and accelerators. These oscillations are driven by two mass-squared differences, solar and atmospheric, at the sub-eV scale. However, longstanding anomalies at shortbaselines might imply the existence of new oscillation frequencies at the eV-scale and the possibility of this sterile state(s) to mix with the three active neutrinos. One of the many future neutrino programs that are expected to provide a final word on this issue is the Short-Baseline Neutrino Program (SBN) at FERMILAB. In this paper, we consider a specific model of Large Extra Dimensions (LED) which provides interesting signatures of oscillation of extra sterile states and we implemented neutrino oscillations as predicted in the LED model in order to perform the sensitivity analysis to the LED parameters at SBN experiment. We have found that SBN is sensitive to the oscillations predicted in the LED model and have the potential to constrain the LED parameter space better than any other oscillation experiment for small values of the absolute lighter neutrino mass and normal ordering.
\end{abstract}

The 20th International Workshop on Neutrinos (NuFact2018)

12-18 August 2018

Blacksburg, Virginia

\footnotetext{
${ }^{*}$ Speaker.

${ }^{\dagger}$ G.V.S is thankful for the support of FAPESP funding Grant No. 2016/00272-9 and No. 2017/12904-2. G.V.S. thanks the useful discussions with Pedro Pasquini and André de Gouvêa.

$\ddagger_{D}$. V. F. is thankful for the support of FAPESP funding Grant No. 2017/01749-6.

$\S^{\S}$ O.L.G.P. is thankful for the support of FAPESP funding Grant No. 2014/19164-6, No. 2016/08308-2, FAEPEX funding grant No. 519.292, CNPQ research fellowship No. 307269/2013-2 and No. 304715/2016-6.
} 


\section{Large Extra Dimensions at the Short Baseline Neutrino Program}

Large Extra Dimension (LED) models postulate that right-handed neutrinos states, which are singlets of the Standard Model gauge group, can also propagate in extra dimensions (bulk) in opposite with SM-charged-fermions that can propagate in the four-dimensional spacetime (brane). The result is a suppression of neutrino Yukawa coupling relative to charged-fermion Yukawa coupling and this could explain the smallness of neutrino masses [1].

The right-handed neutrino fields can be decomposed, in four-dimensions, into massive KaluzaKlein (KK) of infinite towers of sterile neutrinos that modify the neutrino standard oscillation probabilities and change their propagation. One interesting fact is that the eigenstates of KK neutrinos can be written in terms of the three standard neutrino scenario and two extra parameters: the lighter Dirac mass $m_{0}^{D}$ and the compactification radius $R_{E D}$ of the extra dimension. So, we have only two more new parameters to include in the standard oscillation probability. More details are presented in the original paper [2].

\begin{tabular}{||cccc||}
\hline Detector & Active Mass & Distance from BNB target & POT \\
\hline \hline Lar1-ND & $112 \mathrm{t}$ & $110 \mathrm{~m}$ & $6.6 \times 10^{20}$ \\
MicroBooNE & $89 \mathrm{t}$ & $470 \mathrm{~m}$ & $1.32 \times 10^{21}$ \\
ICARUS-T600 & $476 \mathrm{t}$ & $600 \mathrm{~m}$ & $6.6 \times 10^{20}$ \\
\hline
\end{tabular}

Table 1: SBN detector active masses and distances from the local of the neutrino production.

The Short Baseline Neutrino Program (SBN) will align three liquid argon detectors in the central axis of the Booster Neutrino Beam (BNB), located at FERMILAB [3]. Table 1 gives details of the SBN detectors. The goal of SBN includes the ability to resolve a class of experimental anomalies in neutrino physics and to perform the most sensitive search to date for sterile neutrinos at the $\mathrm{eV}$ mass-scale through both appearance and disappearance oscillation channels and this was our motivation to study the LED model at this experiment.

\section{Sensitivity Analysis}

We computed the expected number of events for LED model at the SBN facility by implementing the detectors in the GLoBES [4] c-library. We simulated three years of operation for the neutrino beam in Lar1-ND and ICARUS-T600 detectors and six years in MicroBooNE detector. After event reconstruction, we included an efficiency factor for each channel in order to mimic event rates from the SBN technical draft. The sensitivity curve obtained by SBN in function of the new LED parameters are shown in Figure 1. For comparative reasons, we also show the sensitivity limits from previous analysis in the literature. We can see that the SBN program is sensitive to the LED parameters and this sensitivity is very competitive, respect to other facilities shown in the plot: compare to constraints from other experiments, the SBN sensitivity to the oscillations predicted by the LED mechanism is better than any other limits in the region $m_{0}<2 \times 10^{-1} \mathrm{eV}$, for normal ordering. 

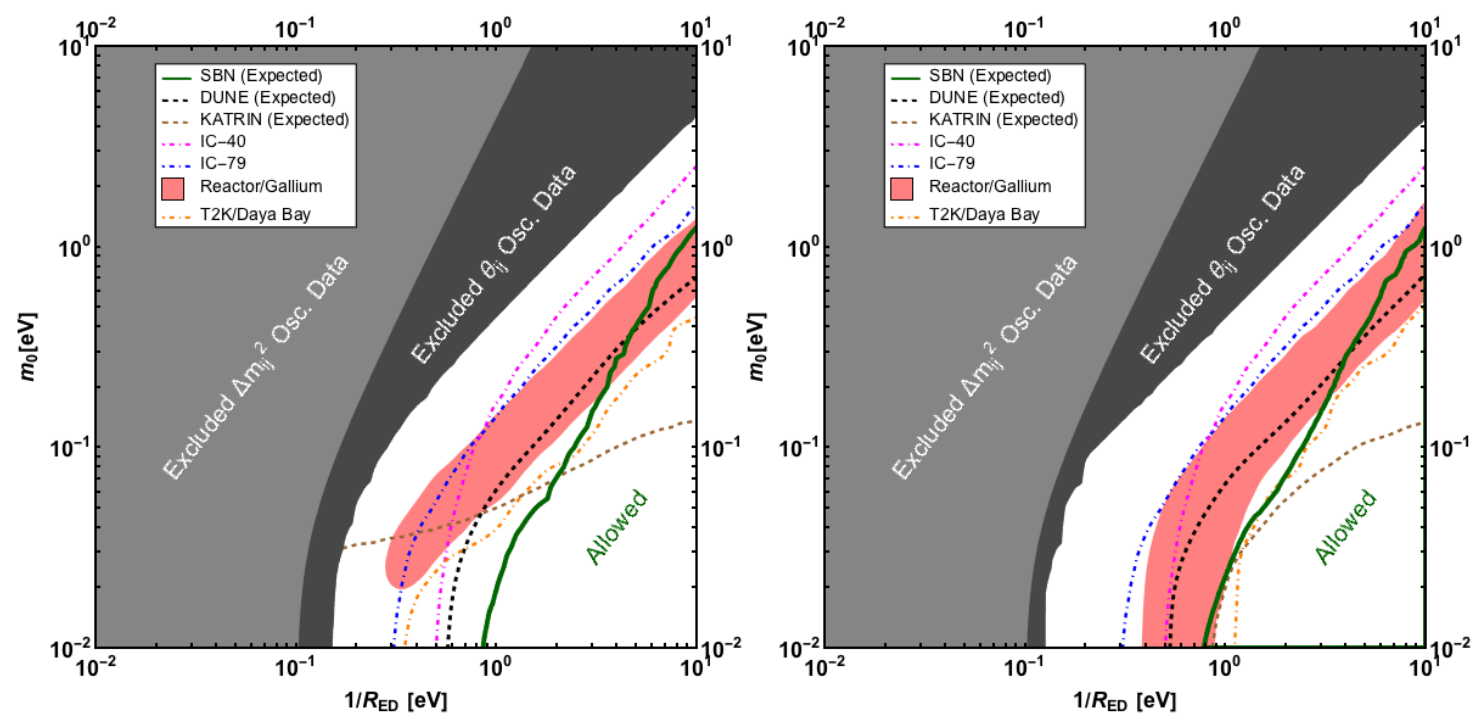

Figure 1: Left (Right) panel: Sensitivity limits for the LED parameters, $R_{\mathrm{ED}}$ and $m_{0}$, considering normal (inverted) ordering of neutrino masses. The regions for LED sensitivity, considering muon disappearance and electron appearance channels, are to the top-left of the curves. Here, we show our $90 \% \mathrm{C}$. L. line from SBN limit (green), the 95\% C. L. lines from DUNE (black) [5], ICECUBE-40 (magenta) and ICECUBE79 (blue) [6], and 95\% C. L. from combined analysis of T2K and Daya Bay (gold) [7]. The 90\% C. L. line from KATRIN sensitivity analysis is also shown (brown) [8] and the pink regions are preferred at $95 \% \mathrm{C}$. L. by the reactor and Gallium anomaly [9]. The light and dark gray regions are excluded due to neutrino oscillation data.

\section{References}

[1] K. R. Dienes, E. Dudas and T. Gherghetta, Nucl. Phys. B 557, 25 (1999) doi:10.1016/S0550-3213(99)00377-6 [hep-ph/9811428].

[2] G. V. Stenico, D. V. Forero and O. L. G. Peres, JHEP 1811, 155 (2018) doi:10.1007/JHEP11(2018)155 [arXiv:1808.05450 [hep-ph]].

[3] M. Antonello et al. [MicroBooNE and LAr1-ND and ICARUS-WA104 Collaborations], arXiv:1503.01520 [physics.ins-det].

[4] P. Huber, J. Kopp, M. Lindner, M. Rolinec and W. Winter, Comput. Phys. Commun. 177, 432 (2007) doi:10.1016/j.cpc.2007.05.004 [hep-ph/0701187].

[5] J. M. Berryman, A. de Gouvêa, K. J. Kelly, O. L. G. Peres and Z. Tabrizi, Phys. Rev. D 94, no. 3, 033006 (2016) doi:10.1103/PhysRevD.94.033006 [arXiv:1603.00018 [hep-ph]].

[6] A. Esmaili, O. L. G. Peres and Z. Tabrizi, JCAP 1412, no. 12, 002 (2014) doi:10.1088/1475-7516/2014/12/002 [arXiv:1409.3502 [hep-ph]].

[7] A. Di Iura, I. Girardi and D. Meloni, J. Phys. G 42, 065003 (2015) doi:10.1088/0954-3899/42/6/065003 [arXiv:1411.5330 [hep-ph]].

[8] V. S. Basto-Gonzalez, A. Esmaili and O. L. G. Peres, Phys. Lett. B 718, 1020 (2013) doi:10.1016/j.physletb.2012.11.048 [arXiv:1205.6212 [hep-ph]].

[9] P. A. N. Machado, H. Nunokawa, F. A. P. dos Santos and R. Z. Funchal, Phys. Rev. D 85, 073012 (2012) doi:10.1103/PhysRevD.85.073012 [arXiv:1107.2400 [hep-ph]]. 